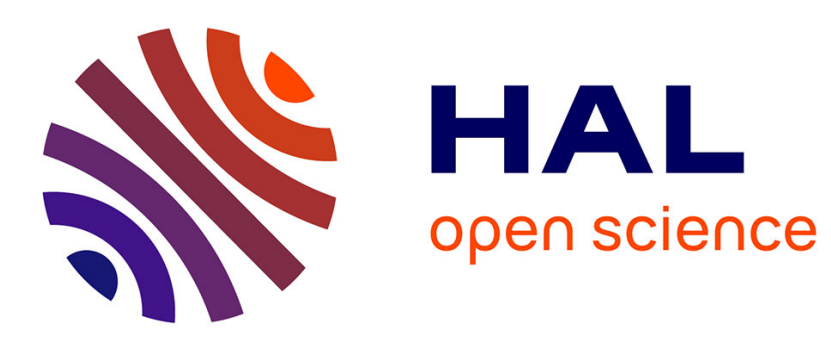

\title{
Quantum modelling of hydrogen chemisorption on graphene and graphite
}

\author{
Frantisek Karlický, Bruno Lepetit, Didier Lemoine
}

\section{To cite this version:}

Frantisek Karlický, Bruno Lepetit, Didier Lemoine. Quantum modelling of hydrogen chemisorption on graphene and graphite. Journal of Chemical Physics, 2014, 140, pp.124702. 10.1063/1.4867995 . hal-00984340

\section{HAL Id: hal-00984340 \\ https://hal.science/hal-00984340}

Submitted on 31 Jan 2017

HAL is a multi-disciplinary open access archive for the deposit and dissemination of scientific research documents, whether they are published or not. The documents may come from teaching and research institutions in France or abroad, or from public or private research centers.
L'archive ouverte pluridisciplinaire HAL, est destinée au dépôt et à la diffusion de documents scientifiques de niveau recherche, publiés ou non, émanant des établissements d'enseignement et de recherche français ou étrangers, des laboratoires publics ou privés. 


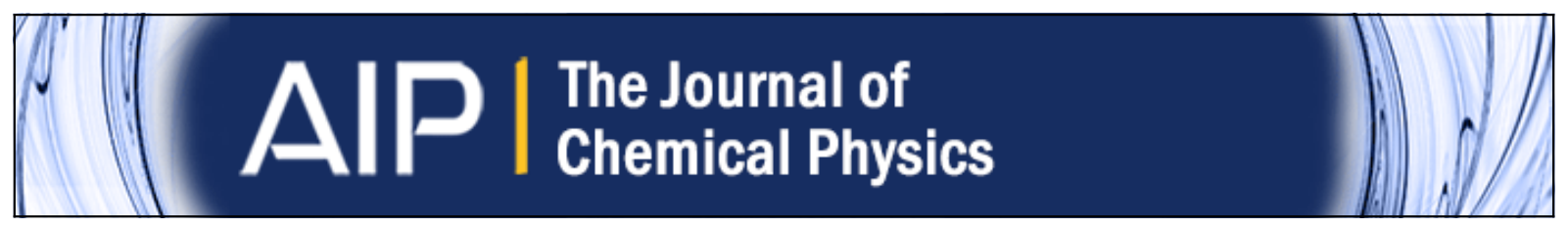

Quantum modelling of hydrogen chemisorption on graphene and graphite

František Karlický, Bruno Lepetit, and Didier Lemoine

Citation: The Journal of Chemical Physics 140, 124702 (2014); doi: 10.1063/1.4867995

View online: http://dx.doi.org/10.1063/1.4867995

View Table of Contents: http://scitation.aip.org/content/aip/journal/jcp/140/12?ver=pdfcov

Published by the AIP Publishing

\section{AlP Re-register for Table of Content Alerts}




\title{
Quantum modelling of hydrogen chemisorption on graphene and graphite
}

František Karlický, ${ }^{1, a)}$ Bruno Lepetit, ${ }^{2, b)}$ and Didier Lemoine ${ }^{2, c)}$

${ }^{1}$ Regional Centre of Advanced Technologies and Materials, Department of Physical Chemistry,

Faculty of Science, Palacký University, Tř. 17. listopadu 12, 77146 Olomouc, Czech Republic

${ }^{2}$ Laboratoire Collisions Agrégats Réactivité, IRSAMC and UMR5589 du CNRS, Université de Toulouse, UPS, 118 route de Narbonne, 31062 Toulouse cedex, France

(Received 29 January 2014; accepted 26 February 2014; published online 24 March 2014)

\begin{abstract}
The chemisorption of hydrogen on graphene or graphite is studied within a quantum formalism involving a subsystem coupled to a phonon bath. The subsystem includes the hydrogen atom approaching the surface perpendicularly right on top of a carbon atom which puckers out of the surface. The bath includes the acoustic and optical phonon modes vibrating perpendicularly to the surface. Couplings between subsystem and bath are obtained with a periodic density functional theory calculation. Trapping probabilities are obtained as a function of the hydrogen atom kinetic energy. These results are discussed in the light of the experimental hydrogenation studies performed on graphite by Zecho et al. [J. Chem. Phys. 117, 8486 (2002)] and on graphene by Haberer et al. [Adv. Mater. 23, 4497 (2011)]. () 2014 AIP Publishing LLC. [http://dx.doi.org/10.1063/1.4867995]
\end{abstract}

\section{INTRODUCTION}

The sticking of hydrogen and other atomic species on graphite and graphene surfaces has been extensively studied over the past decades to gain a better understanding of hydrogen-surface interaction in diverse conditions. For instance, some studies were motivated by the erosion problem of graphite walls interacting with hydrogen plasma in fusion devices. ${ }^{1,2}$ Others contributed to the understanding of the large abundance of molecular hydrogen in the universe as a result of the sticking and subsequent recombination of atomic hydrogen on interstellar dust grains, ${ }^{3-5}$ which are known to have graphitic components. ${ }^{6}$ Another class of motivations for such studies is the prospect of future technological outcomes, like the tuning of the electrical properties of single layer graphene by controlled hydrogenation leading to graphane. ${ }^{7,8}$ Similarly, studies on other species (like oxygen and halogens) also attracted considerable interest over the past few years because of their potential applications, ${ }^{9,10}$ like the recent development of graphene-based nanoelectromechanical systems (NEMS) which can be used as mass sensors. ${ }^{11}$

Sticking, also called adsorption, of hydrogen on carbon surfaces can occur either in the physisorption or in the chemisorption regime. Both processes require that the incoming particle transfers part of its energy to the surface by some dissipative process, in particular phonon excitation. For low energy incoming hydrogen, only physisorption can occur. $\mathrm{H}$ atoms can trap in the shallow potential well, about $40 \mathrm{meV}$ deep ${ }^{12,13}$ resulting from the weak van der Waals interaction between hydrogen and the surface. Several models including phonon excitation showed that this process has a significant probability to occur and is enhanced by diffraction resonances induced by surface corrugation. ${ }^{14-17}$ On the other hand, chemisorption, also called hydrogenation, induces

\footnotetext{
a)Electronic mail: frantisek.karlicky@upol.cz

${ }^{b)}$ Electronic mail: bruno.lepetit@irsamc.ups-tlse.fr

c) Electronic mail: didier.lemoine @irsamc.ups-tlse.fr
}

puckering of the carbon atom closest to the hydrogen, out of the plane of the surface, because of a change in the hybridization of the $\mathrm{C}$ orbitals from $s p^{2}$ to $s p^{3}$. As a result of this lattice distortion, there is a barrier to chemisorption of the order of $0.2 \mathrm{eV}$, as first shown in Refs. 18 and 19. Such chemisorption process was observed experimentally ${ }^{20}$ on highly oriented pyrolitic graphite (HOPG) surfaces at $150 \mathrm{~K}$ exposed to thermal $(2000 \mathrm{~K})$ hydrogen atoms. A sticking coefficient as high as $40 \% \pm 20 \%$ at low H coverage was obtained. More recently, hydrogenation studies for graphene at $300 \mathrm{~K}$ exposed to hydrogen atoms at $3000 \mathrm{~K}$ were performed. ${ }^{21,22}$ The balance between sticking and desorption (associated to $\mathrm{H}_{2}$ recombination) processes yielded $\mathrm{H}: \mathrm{C}$ ratio at saturation coverage equal to 1:4. The measured correlation between time dependent $\mathrm{H}: \mathrm{C}$ stoichiometry and exposure duration provided information on hydrogen adsorption kinetics and an estimate for sticking probability: $P_{\text {ads }} \approx 3.6 \%$. This is significantly smaller than the value obtained by Zecho et al. ${ }^{20}$ The origin of this discrepancy is unclear. As graphite can be considered as a stack of weakly interacting graphene layers, ideal graphite and graphene interact similarly with adsorbing species. ${ }^{17}$ Beyond differences on surface temperature and incoming $\mathrm{H}$ atom energy, surface defects and/or impurities may significantly contribute to the measured discrepancies.

There are also large discrepancies between the different hydrogenation modeling results. The complexity of the problem requires simplifications which induce significant deviations in the resulting adsorption probabilities. In all full quantum models, a separation is performed between a small subsystem in which interactions are strong, containing the $\mathrm{H}$ atom and possibly neighboring $\mathrm{C}$ atoms, and the other $\mathrm{C}$ atoms which play the role of a phonon bath able to dissipate energy from the subsystem. From a wavepacket description of the subsystem-bath interaction, trapping probabilities are obtained. Long enough trapping leads to sticking by further phonon excitation. Sha et al. $^{23}$ performed a realistic model of the subsystem including the $\mathrm{H}$ atom and the carbon atom 
nearest to it but drastically reduced the phonon bath model to a single optical mode. They obtained 5\%-10\% trapped populations and showed that long lived vibrational resonances of the strongly coupled $\mathrm{CH}$ system are important contributors to trapping. This trapping probability is of the same order of magnitude as the experimental result of Haberer et al. ${ }^{21}$ On the other hand, Morisset et al. ${ }^{24,25}$ developed a simplified onedimensional description of the subsystem where the $\mathrm{H}$ atom interacts with the relaxed surface, but used a more realistic description of the phonon bath. Large trapping probabilities of the same order of magnitude as the measurements of Zecho et al. $^{20}$ were obtained. Some or all pieces of the subsystembath model can also be dealt with using classical mechanics. For instance, Kerwin et al. ${ }^{26}$ performed a fully classical treatment of all atoms and obtained trapping probabilities of the order of a few percents. On the fly classical molecular dynamics of Ref. 21 also provided similar small adsorption probabilities. But Morisset et al. ${ }^{27}$ by using a classical description of the phonon bath coupled to their one dimension $\mathrm{H} /$ relaxed surface subsystem, obtained again large trapping probabilities, consistent with the measured values of Zecho et al. ${ }^{20} \mathrm{In}$ addition, a very recent Bohmian trajectory calculation ${ }^{28}$ provided large adsorption probabilities, but strongly dependent on the approximations performed on the quantum corrections to the classical forces, and stressing the importance of quantum effects.

The present contribution extends the series of the full quantum models and mixes the realistic model of the subsystem of Sha et al..$^{23}$ with a realistic model of the phonon bath akin to the one of Morisset et al. ${ }^{24,25}$ Section II describes our implementation of the subsystem-bath separation and of the wavepacket propagation method. Section III gives the computed trapping probabilities as a function of the hydrogen kinetic energy. Our results are discussed in the context of existing experimental and modeling results for graphite and graphene. Section IV summarizes the present work and proposes orientations for future work.

\section{METHODS}

\section{A. Subsystem-bath separation}

Our method relies on a molecular subsystem/bath separation achieved by a Taylor expansion of the interaction potential which depends on the $\mathrm{C}$ atom positions $\left\{\mathbf{R}_{i}\right\}$ ( $i$ is a $\mathrm{C}$ atom label) and on the $\mathrm{H}$ atom one $\mathbf{R}_{\mathrm{H}}$. In our previous studies on physisorption, ${ }^{16,17}$ the Taylor expansion was performed with respect to all $\mathrm{C}$ atom positions, as they are all weakly perturbed by the approach of hydrogen. In the present case, we assume a perpendicular approach of $\mathrm{H}$ atom right on top one $\mathrm{C}$ atom. This singles out this $\mathrm{C}$ atom among the others in the lattice and we call it the central atom in the following. This $\mathrm{C}$ atom interacts strongly with incoming $\mathrm{H}$, puckers out the surface significantly, such that for chemisorption to occur, a new $\mathrm{CH}$ bond is formed, inducing a change in the electronic hybridization in this $\mathrm{C}$ atom from $\mathrm{sp}^{2}$ to $\mathrm{sp}^{3}$ and a large local distortion of the surface, while the other $\mathrm{C}$ atoms are less perturbed (see Sec. III A). We therefore exclude the central $\mathrm{C}$ atom (position labeled $\mathbf{R}_{\mathrm{C}}$ ) from the Taylor expansion such that

$$
\begin{aligned}
V\left(\mathbf{R}_{\mathrm{H}}, \mathbf{R}_{\mathrm{C}},\left\{\mathbf{R}_{i}\right\}\right) \\
=V\left(\mathbf{R}_{\mathrm{H}}, \mathbf{R}_{\mathrm{C}},\{\mathbf{0}\}\right)+\sum_{i} \frac{\partial V\left(\mathbf{R}_{\mathrm{H}}, \mathbf{R}_{\mathrm{C}},\{\mathbf{0}\}\right)}{\partial \mathbf{R}_{i}} \mathbf{u}_{i} \\
\quad+\frac{1}{2} \sum_{i, j} \frac{\partial^{2} V\left(\mathbf{R}_{\mathrm{H}}, \mathbf{R}_{\mathrm{C}},\{\mathbf{0}\}\right)}{\partial \mathbf{R}_{i} \partial \mathbf{R}_{j}} \mathbf{u}_{i} \mathbf{u}_{j},
\end{aligned}
$$

where $\left\{\mathbf{u}_{i}\right\}$ gives the set of displacements of $\mathrm{C}$ atoms \# $i$ away from equilibrium positions (denoted by $\{\mathbf{0}\}$ ). The summation indices $i$ and $j$ exclude the central $\mathrm{C}$ atom. We assume that the crossed derivatives between atoms \# $i$ and $j$ depend weakly on the positions of the central $\mathrm{C}$ and $\mathrm{H}$ atoms: $\frac{\partial^{2} V\left(\mathbf{R}_{\mathrm{H}}, \mathbf{R}_{\mathrm{C}},\{\mathbf{0}\}\right)}{\partial \mathbf{R}_{i} \partial \mathbf{R}_{j}}=\frac{\partial^{2} V\left(\mathbf{R}_{\mathrm{H}}=\infty, \mathbf{R}_{\mathrm{C}}=\mathbf{0},\{\mathbf{0}\}\right)}{\partial \mathbf{R}_{i} \partial \mathbf{R}_{j}}$ and that graphene vibrations ( $\mathrm{H}$ atom infinitely far) can be described by an harmonic potential. Then, setting apart in the bath potential the terms coupling the central $\mathrm{C}$ atom to the others, the last term of Eq. (1) can be written:

$$
\begin{aligned}
\frac{1}{2} \sum_{i, j} & \frac{\partial^{2} V\left(\mathbf{R}_{\mathrm{H}}, \mathbf{R}_{\mathrm{C}},\{\mathbf{0}\}\right)}{\partial \mathbf{R}_{i} \partial \mathbf{R}_{j}} \mathbf{u}_{i} \mathbf{u}_{j} \\
= & V\left(\mathbf{R}_{\mathrm{H}}=\infty, \mathbf{R}_{\mathrm{C}},\left\{\mathbf{u}_{i}\right\}\right) \\
& -\frac{1}{2} \frac{\partial^{2} V\left(\mathbf{R}_{\mathrm{H}}=\infty, \mathbf{R}_{\mathrm{C}}=\mathbf{0},\{\mathbf{0}\}\right)}{\partial \mathbf{R}_{\mathrm{C}}^{2}} \mathbf{u}_{\mathrm{C}}^{2} \\
& -\frac{1}{2} \sum_{i} \frac{\partial^{2} V\left(\mathbf{R}_{\mathrm{H}}=\infty, \mathbf{R}_{\mathrm{C}}=\mathbf{0},\{\mathbf{0}\}\right)}{\partial \mathbf{R}_{\mathrm{C}} \partial \mathbf{R}_{i}} \mathbf{u}_{\mathrm{C}} \mathbf{u}_{i} .
\end{aligned}
$$

We neglect the second term (quadratic in $\mathbf{u}_{\mathrm{C}}$ ) on the righthand side of Eq. (2), as it is only one among (infinitely) many contributors to the bath harmonic potential. Then using $\frac{\partial^{2} V\left(\mathbf{R}_{\mathbf{H}}=\infty, \mathbf{R}_{\mathrm{C}}=\mathbf{0},\{\mathbf{0}\}\right)}{\partial \mathbf{R}_{C} \partial \mathbf{R}_{i}} \mathbf{u}_{\mathrm{C}}=\frac{\partial V\left(\mathbf{R}_{\mathbf{H}}=\infty, \mathbf{R}_{\mathrm{C}},\{\mathbf{0}\}\right)}{\partial \mathbf{R}_{i}}$, we can simplify Eq. (1) into

$$
\begin{aligned}
V\left(\mathbf{R}_{\mathrm{H}}, \mathbf{R}_{\mathrm{C}},\left\{\mathbf{R}_{i}\right\}\right) \\
=V\left(\mathbf{R}_{\mathrm{H}}, \mathbf{R}_{\mathrm{C}},\{\mathbf{0}\}\right)+V\left(\mathbf{R}_{\mathrm{H}}=\infty, \mathbf{R}_{\mathrm{C}},\left\{\mathbf{R}_{i}\right\}\right) \\
\quad+\sum_{i}\left(\frac{\partial V\left(\mathbf{R}_{\mathrm{H}}, \mathbf{R}_{\mathrm{C}},\{\mathbf{0}\}\right)}{\partial \mathbf{R}_{i}}-\frac{\partial V\left(\mathbf{R}_{\mathrm{H}}=\infty, \mathbf{R}_{\mathrm{C}},\{\mathbf{0}\}\right)}{\partial \mathbf{R}_{i}}\right) \mathbf{u}_{i} .
\end{aligned}
$$

This defines our partition of the potential into subsystem, bath, and coupling terms.

Because we assume perpendicular hydrogen approach, the acoustic (ZA) and optical (ZO) phonon modes perpendicular to the surface are expected to be the most strongly coupled to the incoming $\mathrm{H}$ atom motion. This justifies the approximation performed to exclude from our model the longitudinal and transverse modes parallel to the surface but to retain the ZA and ZO bands. Adding these longitudinal and transverse in-plane vibrational modes would increase the density of states contributing to dissipation and presumably the efficiency of the process, but in a limited amount due to weak coupling between in-plane vibration and perpendicular $\mathrm{H}$ atom approach. Each $\mathrm{C}$ atom and $\mathrm{H}$ atom can thus be described by a single coordinate $z_{i}, z_{\mathrm{C}}$ or $z_{\mathrm{H}}$ describing its height with respect to the surface plane. Introducing Jacobi coordinates: $z=z_{\mathrm{H}}-z_{\mathrm{C}}$ and $Z=\frac{m_{\mathrm{H}} z_{\mathrm{H}}+m_{\mathrm{C}} z_{\mathrm{C}}}{M}$ with $M=m_{\mathrm{H}}+m_{\mathrm{C}}$ 
( $m_{\mathrm{H}}$ and $m_{\mathrm{C}}$ are $\mathrm{H}$ and $\mathrm{C}$ atom masses), the total Hamiltonian can be partitioned as: $H=H_{\mathrm{s}}+H_{\mathrm{b}}+C$, where

$$
H_{\mathrm{s}}=-\frac{\hbar^{2}}{2 M} \frac{\partial^{2}}{\partial Z^{2}}-\frac{\hbar^{2}}{2 m} \frac{\partial^{2}}{\partial z^{2}}+V(Z, z,\{\mathbf{0}\}),
$$

( $m$ : $\mathrm{CH}$ reduced mass) and $H_{\mathrm{b}}$ is the bath Hamiltonian describing the vibrations of the graphene harmonic lattice. Both Hamiltonians are coupled by the term $C\left(Z, z,\left\{z_{i}\right\}\right)$ $=-\sum_{i} F_{i}(Z, z) z_{i}$, where the force exerted by the $\mathrm{CH}$ subsystem on lattice atom \# $i$ is given by

$$
F_{i}(Z, z)=-\frac{\partial V\left(z_{\mathrm{H}}, z_{\mathrm{C}},\{0\}\right)}{\partial z_{i}}+\frac{\partial V\left(z_{\mathrm{H}}=\infty, z_{\mathrm{C}},\{0\}\right)}{\partial z_{i}} .
$$

The second term on the right-hand side of Eq. (5) is a correction to the force thanks to which the subsystem-bath coupling vanishes when the $\mathrm{H}$ atom is at large distance.

\section{B. Wavepacket method}

The dynamics is described by a wavepacket associated to the total Hamiltonian. ${ }^{29,30}$ It is expanded on a basis of resonant and phonon states:

$$
\begin{aligned}
& \psi\left(Z, z,\left\{z_{i}\right\}, t\right) \\
& \quad=\sum_{j=1-N_{r}, \lambda=0, \pm 1, \sigma=1-2, k=1-N_{p}} c_{j}^{\lambda \sigma k}(t) \varphi_{j}(Z, z)\left|\{n\}_{\lambda \sigma \mathbf{Q}_{k}}\right\rangle .
\end{aligned}
$$

The summation index $j$ runs over the $N_{r}$ lowest energy 2 dimensional eigenstates $\varphi_{j}(Z, z)$ of $H_{s}$, the corresponding eigenvalues are labeled $e_{j} . \sigma$ identifies the ZA and ZO bands. $k$ labels the $N_{p}$ phonon wavevectors $\mathbf{Q}_{k}$ which define a discretization of the first Brillouin zone of graphene. The initial vibrational state of graphene, when $\mathrm{H}$ is far from the surface, corresponds to $\lambda=0$. The initial set of occupation numbers of the different phonon modes at frequency $\omega_{\sigma \mathbf{Q}_{k}}$ is assumed to be the Bose-Einstein distribution at surface temperature $T_{s}$ : $n_{\lambda=0 \sigma \mathbf{Q}_{k}}=\left(e^{\frac{\hbar \omega_{\sigma} \mathbf{Q}_{k}}{k T_{s}}}-1\right)^{-1}$. By interaction with the $\mathrm{CH}$ subsystem, the bath can excite $(\lambda=+1)$ or de-excite $(\lambda=-1)$ by creating or annihilating a phonon in band $\sigma$ with momentum $\mathbf{Q}_{k}$. The final bath state occupation numbers $\{n\}_{\lambda \sigma} \mathbf{Q}_{k}$ are thus the same as the initial ones $\{n\}_{\lambda=0 \sigma \mathbf{Q}_{k}}$, except for the mode $\sigma$, $\mathbf{Q}_{k}$ for which final occupation is different from the initial one by $\lambda$.

As the expansion of the wave-function is thus truncated to bath states different from the initial one by a single phonon, processes involving simultaneous or sequential creation or annihilation of several phonons are not described by this formalism. This severe truncation of the phonon basis is necessary to confine the numerical simulation within tractable limits. It allows also to make comparisons with the previous quantum models which assume similar truncation. ${ }^{24,25}$

The $N_{r}\left(4 N_{p}+1\right)$ coefficients $c_{j}^{\lambda \sigma k}(t)$ in Eq. (6) are solutions of a set of coupled differential equations:

$$
i \hbar \frac{d c_{j}^{\lambda \sigma k}(t)}{d t}=\left(e_{j}+\lambda \hbar \omega_{\sigma k}\right) c_{j}^{\lambda \sigma k}(t)+\sum_{j^{\prime}, \lambda^{\prime}, \sigma^{\prime}, k^{\prime}} C_{\lambda^{\prime} \sigma^{\prime} k^{\prime} j^{\prime}}^{\lambda \sigma k j} c_{j^{\prime}}^{\lambda^{\prime} \sigma^{\prime} k^{\prime}}(t) .
$$

The coupling matrix elements: $C_{\lambda^{\prime} \sigma^{\prime} k^{\prime} j^{\prime}}^{\lambda \sigma k j}=-\sum_{i}\left\langle\varphi_{j}\left|F_{i}\right| \varphi_{j^{\prime}}\right\rangle$ $\left\langle\{n\}_{\lambda \sigma \mathbf{Q}_{k}}\left|z_{i}\right|\{n\}_{\lambda^{\prime} \sigma^{\prime} \mathbf{Q}_{k}^{\prime}}\right\rangle$ are nonzero only when $\lambda=0$ or $\lambda^{\prime}$ $=0$. They can be simplified further by expanding the surface displacements $z_{i}$ over phonon modes,

$$
\begin{aligned}
& \left\langle\{n\}_{\lambda=0 \sigma \mathbf{Q}_{k}}\left|z_{i}\right|\{n\}_{\lambda^{\prime} \sigma^{\prime} \mathbf{Q}_{k}^{\prime}}\right\rangle \\
& =\left(\frac{\hbar}{2 N_{p} m_{\mathrm{C}} \omega_{\sigma^{\prime} \mathbf{Q}_{k}^{\prime}}}\right)^{\frac{1}{2}} e^{i \mathbf{Q}_{k}^{\prime} \mathbf{R}_{i}} \epsilon_{\sigma^{\prime} \mathbf{Q}_{k}^{\prime}}^{i}\left(n_{\lambda=0 \sigma^{\prime} \mathbf{Q}_{k}^{\prime}}+\delta_{\lambda^{\prime} 1}\right)^{\frac{1}{2}},
\end{aligned}
$$

where $\epsilon_{\sigma \mathbf{Q}_{k}}^{i}$ is the phonon polarization vector associated to $\mathrm{C}$ atom $\# i$. The Kronecker symbol $\delta_{\lambda^{\prime} 1}$ distinguishes phonon excitation from annihilation. Integration of these coupled equations provides time dependent total populations associated to the different surface states $\lambda=0, \pm 1$ :

$$
P_{t o t}^{\lambda}(t)=\sum_{\sigma k j}\left|c_{j}^{\lambda \sigma k}(t)\right|^{2}
$$

Trapped populations in the chemisorption well bounded by a maximum $\mathrm{CH}$ distance $z_{\max }$ can be defined for each surface state as:

$$
P_{\text {trap }}^{\lambda}(t)=\sum_{\sigma k} \iint^{z_{\max }} d Z d z\left|\sum_{j} c_{j}^{\lambda \sigma k}(t) \varphi_{j}(Z, z)\right|^{2},
$$

as well as untrapped ones:

$$
P_{\text {untrap }}^{\lambda}(t)=P_{\text {tot }}^{\lambda}(t)-P_{\text {trap }}^{\lambda}(t) .
$$

\section{Computational implementation}

The calculations of the flat frozen $\mathrm{H}-\mathrm{C}$-graphene interaction potential $V(Z, z,\{\mathbf{0}\})$ (Eq. (4)) and forces $F_{i}(Z, z)$ (Eq. (5)) were performed using the spin-polarized version of density functional theory (DFT) in the generalized gradient approximation (GGA) of Perdew-Burke-Ernzerhof (PBE). ${ }^{31}$ The $a b$ initio total-energy and molecular dynamics program VASP (Vienna $a b$ initio simulation program) developed at the Institut für Materialphysik of the Universität Wien has been used. ${ }^{32}$ The interactions between the ionic cores and the electrons are described by fully nonlocal optimized projector augmented-wave (PAW) potentials. ${ }^{33}$ A $4 \times 4$ supercell (containing 32 carbon atoms) was chosen, see Fig. 1 , and $5 \times 5$ $\times 1 \mathrm{k}$-points (including the $\Gamma$ point) sampled the first Brillouin zone. As periodic boundary conditions were applied in all three dimensions, a vacuum layer of $20 \AA$ was included to minimize any (spurious) interaction between adjacent layers. A cut-off energy of $500 \mathrm{eV}$ was applied for the plane-wave basis set. An optimized unit cell of graphene with lattice constant $a=2.468 \AA$ was obtained. This value was kept to describe the frozen graphene lattice when $\mathrm{H}$ and central $\mathrm{C}$ atom are displaced. Potential energies and forces were calculated for a $2 \mathrm{D}$ rectangular equidistant grid of $z_{\mathrm{H}} \in[0.8,0.9,1.0, \ldots$, $3.3,3.4] \AA$ and $z_{\mathrm{C}} \in[-0.40,-0.35, \ldots, 0.75,0.80] \AA$, i.e., total $28 \times 25=675$ points were evaluated. $2 \mathrm{D}$ cubic splines were used to interpolate potential and forces for any $\mathrm{CH}$ positions. However, because of $C_{3}$ rotational symmetry of the lattice with respect to central $\mathrm{C}$ and $\mathrm{H}$ atoms, atoms located on rings centered on these atoms experience the same force perpendicularly to the graphene plane (see Fig. 1). Four rings, 

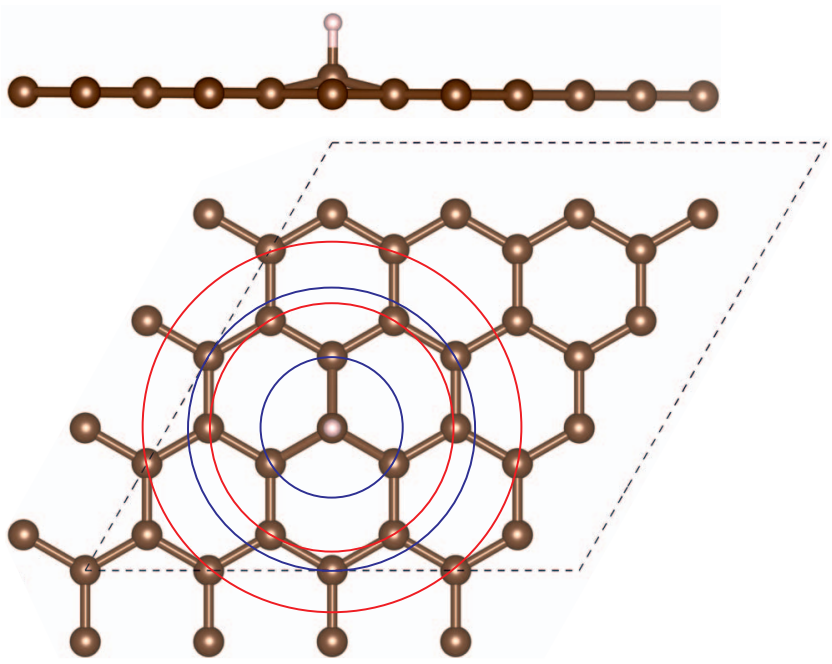

FIG. 1. Graphene $4 \times 4$ supercell and central $\mathrm{H}$ atom used in the potential $V(Z, z,\{\mathbf{0}\})$ (Eq. (4)) and force calculation $F_{i}(Z, z)($ Eq. (5)). The graphene lattice constant is optimized without $\mathrm{H}$ atom and frozen at the resulting value, $2.468 \AA$, when $\mathrm{H}$ and central $\mathrm{C}$ atoms are displaced. The four circles indicate the four rings of atoms which interact with the central $\mathrm{CH}$ system in our calculation.

rings \# 1 and 3 including 3 atoms, rings \# 2 and 4 including 6 ones, were included in the calculation. Extra rings are not necessary as our DFT calculations indicates that forces die off sharply beyond ring \# 4 .

The semi empirical valence force field model of Aizawa et al. ${ }^{34}$ was used for the harmonic phonon bath potential (term $V\left(\mathbf{R}_{\mathrm{H}}=\infty, \mathbf{R}_{\mathrm{C}},\left\{\mathbf{R}_{i}\right\}\right)$ in Eq. (3)). This model relies on two spring constants describing changes in the potential due to the deformation of the surface. One characterizes the displacement of each carbon atom out of the plane formed by its three neighbors $(\gamma=2.11 \mathrm{eV})$ and the other one describes twisting $(\delta=1.98 \mathrm{eV})$, which induces a force similar to that making ethylene flat. This model has accurately reproduced measured phonon dispersion curves ${ }^{34}$ and is in excellent agreement with first-principles calculations. ${ }^{35}$

It is known that thermal fluctuations destroy order in the two dimensional graphene sheet. ${ }^{36,37}$ Flat graphene can be stabilized with respect to these thermal fluctuations, either by having it supported on a substrate, for instance a silicon oxide layer, or by suspending it on holes in such substrate. ${ }^{17}$ The weak van der Waals interaction energy between graphene and $\mathrm{SiO}_{2}$ substrate has been measured to be $\approx 0.1 \mathrm{~J} / \mathrm{m}^{2}$ (Ref. 38) for an average separation of $\approx 0.42 \mathrm{~nm} .{ }^{39}$ In the harmonic approximation, this corresponds to a force constant $k^{\text {sub }}$ $=0.4 \mathrm{~N} / \mathrm{m}$ linking each carbon atom to the substrate ${ }^{38}$ This value was used in our calculation. Taking advantage of symmetry, the 1st Brillouin zone was reduced by a factor 12 to the $\Gamma$-K-M triangle, which was discretized with $N_{p}=43$ k-points for the two bands, $\mathrm{ZA}$ and $\mathrm{ZO}$.

The number of resonances $\varphi_{j}(Z, z)$ used in expansion of the wavefunction (Eq. (6)) was $N_{r}=648$. It spans the spectral range $[-0.42,1.5] \mathrm{eV}$. These resonances were obtained on a grid of 121 points $z \in[0.4,5.8] \AA$ and 61 points $Z$ $\in[-0.4,1.22] \AA$. The use of this optimized basis provides a much more compact representation of the wavefunction than the one which would be obtained by keeping the primitive 121 $\times 61=7381$ Discrete Variable Representation (DVR) basis. Its use limits the total number of coupled equations (Eq. (7)) to $N_{r}\left(4 N_{p}+1\right)=112014$.

The surface temperature is $T_{s}=300 \mathrm{~K}$. The initial wavepacket is centered in the asymptotic region around $z=3.2 \AA$. Due to its energy width $82 \mathrm{meV}$, it is confined in the integration box. This width also accounts for some unavoidable energy spreading in the experimental $\mathrm{H}$ beam. ${ }^{21,22}$ Central energy of the wavepacket in varied in the range 0.1$0.8 \mathrm{eV}$. A Crank-Nicholson algorithm was used to integrate the coupled differential (Eq. (7)) with a time step $0.72 \mathrm{fs}$ up to $80 \mathrm{fs}$. This time limit is chosen to discard unphysical trapping which occurs at later times for the component of the wavepacket reflecting at the edge of the spatial grid, where no absorbing potential has been placed. We showed in Ref. 16 that such an algorithm is well suited for the banded structure of the coupling matrix considered here. Finally, the upper bound for the trapping region (Eq. (10)) is chosen as $z_{\max }=1.85 \AA$, which corresponds the $\mathrm{CH}$ distance for the saddle point associated to chemisorption.

\section{RESULTS AND DISCUSSIONS}

\section{A. Potential energy surface and forces}

Since the first calculation by Jeloaica et al. ${ }^{18}$ portions of the potential energy surface (PES) for H-graphite interaction have been computed several times (see for instance Refs. 19, 40-54). Part of these results are compiled in Ref. 50. A fit of the PES has been developed. ${ }^{26}$ All the calculations show a minimum for chemisorption when the atom nearest to the approaching $\mathrm{H}$ is allowed to pucker out of the surface and to produce a barrier to chemisorption. The validity of these DFT results was confirmed by a recent comparison with a set of ab initio coupled-cluster calculations. ${ }^{55}$ Calculations were usually performed allowing for full relaxation of all atoms of the model. The chemisorption minimum energy was found to be $0.79 \mathrm{eV},{ }^{50} 0.76 \mathrm{eV}^{43}$ and $0.85 \mathrm{eV}^{45}$ for the same $4 \times 4$ supercell as the one used here. The central $\mathrm{C}$ atom puckers out of the surface by $z_{\mathrm{C}}=0.48 \AA .{ }^{50} \mathrm{Un}$ der similar fully relaxed conditions, we checked that we obtain a chemisorption energy of $0.809 \mathrm{eV}$ fully consistent with these previous results. However, our formalism (see for instance Eq. (4)) requires frozen carbon atoms at equilibrium except for the central one. Under such constraints, we find a slightly shallower minimum $(0.613 \mathrm{eV})$ and less puckering of the $\mathrm{C}$ atom $\left(z_{\mathrm{C}}=0.35 \AA\right)$ for $z_{\mathrm{H}}=1.5 \AA$. The resulting PES surface is shown on Fig. 2. These differences are related to the lower degree of relaxation allowed in our calculation. It was shown by Bachellerie et al. ${ }^{52}$ that the central $\mathrm{C}$ atom is not the only one to pucker out of the surface when an $\mathrm{H}$ atom approaches. The $\mathrm{C}$ atoms around the central one contribute to a hillock structure at the fully relaxed equilibrium configuration. In their calculation, ${ }^{52}$ this configuration corresponds to a chemisorption energy of $0.72 \mathrm{eV}$, the central atom puckers out by $0.48 \AA$, the first ring surrounding $\mathrm{C}$ atoms by $0.13 \AA$, the second and third ones by $0.06 \AA$, for an optimal approach of the $\mathrm{H}$ atom: $z_{\mathrm{H}}=1.6 \AA$. Constraining these surrounding atoms to remain fixed on the initial graphene plane reduces 


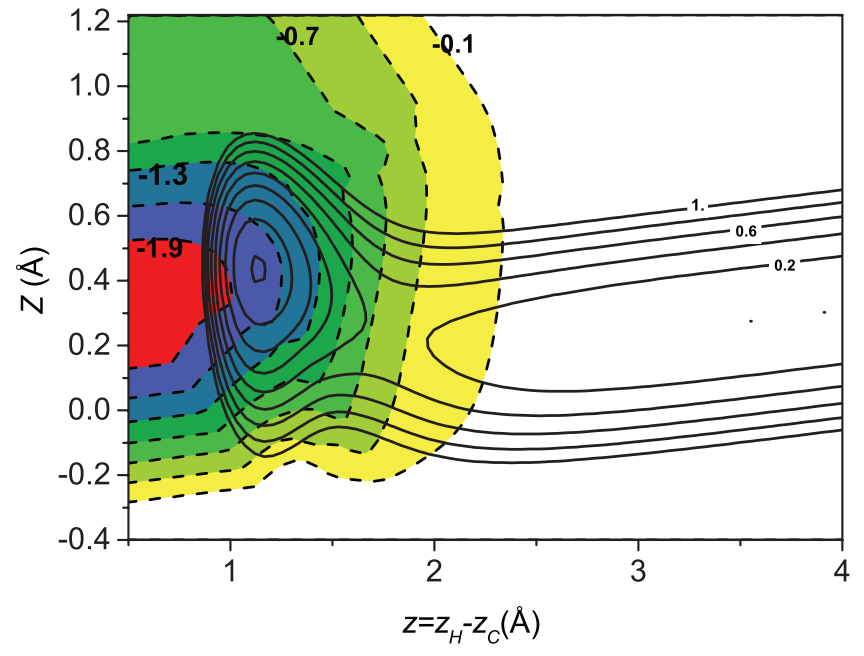

FIG. 2. Full lines: Contours of the $2 \mathrm{D}$ potential energy surface describing the interaction between a graphene layer and an $\mathrm{H}$ atom at height $z_{H}$ in a perpendicular approach right on top of the central $\mathrm{C}$ atom, located at height $z_{C}$. The coordinate $z$ is the $\mathrm{CH}$ distance, and $Z$ stands for the height of the $\mathrm{CH}$ center of mass with respect to the unperturbed surface. Adjacent contour levels are separated by $0.2 \mathrm{eV}$. Dashed lines: Contours of the corrected force (Eq. (5)) perpendicular to the graphene plane exerted on the first ring of $\mathrm{C}$ atoms and induced by the approaching $\mathrm{H}$ atom. Adjacent contour levels are separated by $0.3 \mathrm{eV} / \AA$.

the chemisorption energy and the central atom puckering. Notice however that the puckering of the central atom relative to the first ring ones is the same in their fully relaxed calculation $(0.48-0.13=0.35 \AA)$ and ours $(0.35-0.0=0.35 \AA)$. Our calculation also provides a barrier to chemisorption of $0.23 \mathrm{eV}$ for the configuration: $z_{\mathrm{H}}=1.9 \AA, z_{\mathrm{C}}=0.1 \AA$, which is consistent with previous calculations and experiments. ${ }^{20}$

Figure 3 shows the uncorrected force $F_{i}^{\text {un }}$ $=-\frac{\partial V\left(z_{\mathrm{H}}, z_{\mathrm{C}},\{0\}\right)}{\partial z_{i}}$ for the atoms located on ring \# 1 in the $\left(z_{\mathrm{C}}, z_{\mathrm{H}}\right)$ coordinate system. The sign convention is such that the attractive forces pointing toward the $\mathrm{H}$ atom side are positive. When $\mathrm{H}$ is very far, the force depends almost

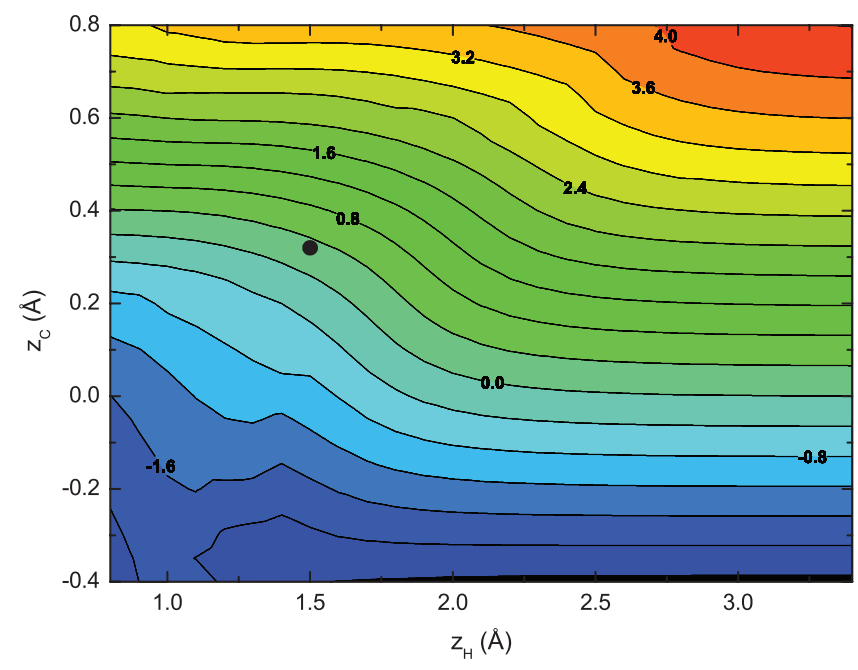

FIG. 3. Component of the force (in eV/Å) perpendicular to the graphene plane on the first ring atoms as a function of the central $\mathrm{C}$ atom and $\mathrm{H}$ atom positions (in $\AA$ ). The dot indicates the location of the minimum of the chemisorption potential well. linearly on $z_{\mathrm{C}}$, the corresponding force constant estimated from the slope of this linear dependence is $5.97 \mathrm{eV} / \AA^{2}$. For such configurations where $\mathrm{H}$ interaction can be neglected, the Aizawa model ${ }^{34}$ provides estimates of the forces resulting from the distortion of the lattice. The central atom experiences $F_{\mathrm{C}}^{A}=-\frac{36 \gamma+6 \delta}{a^{2}} z_{\mathrm{C}}$ and for the ring $\# i$ atoms $(i$ $=1-4$ ), we have: $F_{1}^{A}=\frac{18 \gamma}{a^{2}} z_{\mathrm{C}}, F_{2}^{A}=\frac{-3 \gamma+\delta}{a^{2}} z_{\mathrm{C}}, F_{3}^{A}=\frac{2 \delta}{a^{2}} z_{\mathrm{C}}$, and $F_{4}^{A}=-\frac{\delta}{a^{2}} z_{\mathrm{C}}$. The forces on the more distant atoms are exactly 0 in this model. The force constant associated to the first ring is $\frac{18 \gamma}{a^{2}}=5.94 \mathrm{eV} / \AA^{2}$, in good agreement with the estimate from the DFT calculation.

As $z_{\mathrm{H}}$ is decreased from large values with a frozen and flat $\left(z_{\mathrm{C}}=0\right)$ graphene surface, an attractive force builds up on the central atom, as seen from the gradient of the potential of Fig. 2. This force is partly counterbalanced by a repulsive one on the ring \# 1 atoms, as seen on Fig. 3. If we now allow the central atom to pucker out toward $\mathrm{H}$ (fixed for instance at $z_{\mathrm{H}}$ $=1.5 \AA$ ), the force on the first ring atoms increases approximately linearly with $z_{\mathrm{C}}$ and changes sign for $z_{\mathrm{C}}=0.27 \AA$. The force constant which is the slope of this linear increase is $5.41 \mathrm{eV} / \AA^{2}$, which is only $10 \%$ different from the value obtained for distant $\mathrm{H}$. This indicates that the presence of the $\mathrm{H}$ atom does not strongly perturb the carbon-carbon force constant. Increasing $z_{\mathrm{C}}$ further to the equilibrium chemisorption position $z_{\mathrm{C}}=0.35 \AA$ thus provides a positive uncorrected force, as it should be to allow for extended puckering. At chemisorption equilibrium, the forces experienced by the ring atoms are given the DFT calculation as: $F_{1}^{\text {un }}=0.44, F_{2}^{\text {un }}$ $=-0.18, F_{3}^{\text {un }}=0.22$, and $F_{4}^{\text {un }}=-0.15 \mathrm{eV} / \AA$. They are negligible for the more distant rings. As the alternation of signs between rings is reminiscent of a similar one observed on the Aizawa forces for distant $\mathrm{H}$, it is related to the stiffness of the graphene sheet. However, the Aizawa model which does not include $\mathrm{H}$ perturbation particularly important for the closest rings obviously cannot give quantitative predictions of these forces.

The corrected force $F_{1}(Z, z)$ (see Eq. (5)) is shown on Fig. 2 for the first ring. It corresponds in fact to the difference, which obviously vanishes at large $z_{\mathrm{H}}$, between forces at finite and asymptotic $z_{\mathrm{H}}$ values. Physically, this singles out the direct effect of the presence $\mathrm{H}$ atom from the indirect one mediated by the puckering of the central $\mathrm{C}$ atom. As both forces at finite and asymptotic distances have an approximate linear dependence in $z_{\mathrm{C}}$ with comparable slopes, their difference $F_{1}(Z$, $z$ ) is expected to have a weak dependence in $Z\left(\approx_{z_{C}}\right)$, as seen from the fact that contour lines are almost vertical on Fig. 2 in the vicinity of the minimum energy path to chemisorption. The direct effect of the $\mathrm{H}$ atom perturbation is clearly a repulsive force on the first ring becoming more intense as the $\mathrm{H}$ atom approaches.

\section{B. Quantum dynamics}

Figure 4 shows trapped populations $P_{\text {trap }}^{\lambda}(t)$ (Eq. (10)) as well as untrapped ones $P_{\text {untrap }}^{\lambda}(t)$ (Eq. (11)) for an incoming $\mathrm{H}$ atom wavepacket centered around $0.38 \mathrm{eV}$ and for different surface states: either without $(\lambda=0)$, or with surface state change, i.e., excitation $(\lambda=1)$ or annihilation 


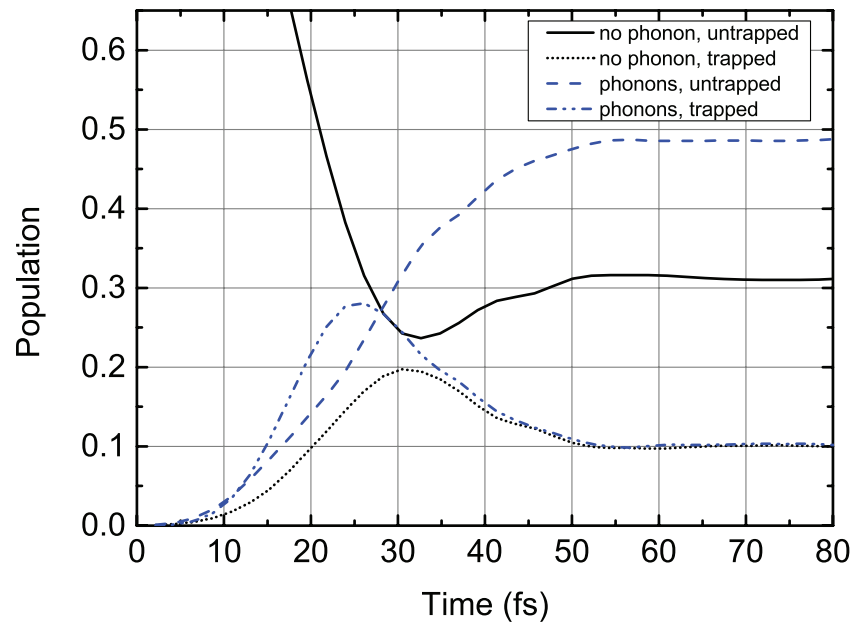

FIG. 4. Time dependence of different populations describing the H-graphene interaction. The incoming $\mathrm{H}$ atom is described by a wavepacket with $0.38 \mathrm{eV}$ mean energy and $81 \mathrm{meV}$ energy width. "Phonon" and "no phonon" labels refer respectively to the $\lambda= \pm 1$ (phonon excitation/annihilation) and $\lambda=0$ (no phonon interaction) components of the wavefunction. For each of these components, "trapped" and "untrapped" refer to the populations corresponding to $\mathrm{C}-\mathrm{H}$ distance respectively smaller and larger than $1.85 \AA$, the saddle point location. Surface temperature is $300 \mathrm{~K}$.

$(\lambda=-1)$ of phonons. At short times, only the $P_{\text {untrap }}^{\lambda=0}(t)$ is nonzero, as the corresponding subspace includes entirely the initial wavepacket. This population decreases and the others increase when the incoming $\mathrm{H}$ atom collides with the surface. The phonon excitation process is fast, trapped populations with or without surface state change increase with comparable rates. Surface state change is irreversible in our model, but trapped populations, even in the case of surface state change, can decrease as the $\mathrm{H}$ atom moves away. Interestingly, a significant part $(\approx 10 \%)$ of the initial population remains trapped in the chemisorption well, for both modified and unmodified surface states. These trapped populations may eventually become stuck to the surface by subsequent phonon emissions not included in our model. These trapped populations are visible at times later than $50 \mathrm{fs}$ on Fig. 4. We thus obtain a total trapping probability $P_{\text {trap }}=\sum_{\lambda} P_{\text {trap }}^{\lambda}(t \rightarrow \infty)$ $=20 \%$ for $0.38 \mathrm{eV}$ collision energy.

It was shown in Ref. 23 that such trapping is related to the population of long lived resonances associated to the vibrational motion of the $\mathrm{CH}$ group on the surface. These resonances have been classified with 2 quantum numbers $(n, N)$ associated with the vibrational modes along $z$ and $Z$, namely, $\mathrm{CH}$ and $\mathrm{C}$-lattice stretch modes. It was shown that the lifetime of these resonances is strongly dependent on the excitation level of these modes. At a given energy, increasing $\mathrm{CH}$ stretch excitation from $n=0$ to $n=1$ decreases lifetime by 2 or 3 orders of magnitude, from $n=1$ to $n=2$ by 1 or 2 orders of magnitude. By contrast, increasing C-lattice excitation $N$ by 1 decreases lifetime by less than 1 order of magnitude. Only $n=0$ and $n=1$ states have lifetimes higher than $100 \mathrm{fs}$ in the energy range considered here. Only these states can contribute to trapping at the time scale considered here $(\leq 80 \mathrm{fs})$, as $n>1$ states decay too quickly at this time scale. However, as $n=0$ resonances are longer lived states and thus have narrower band widths than $n=1$ ones, they are less populated

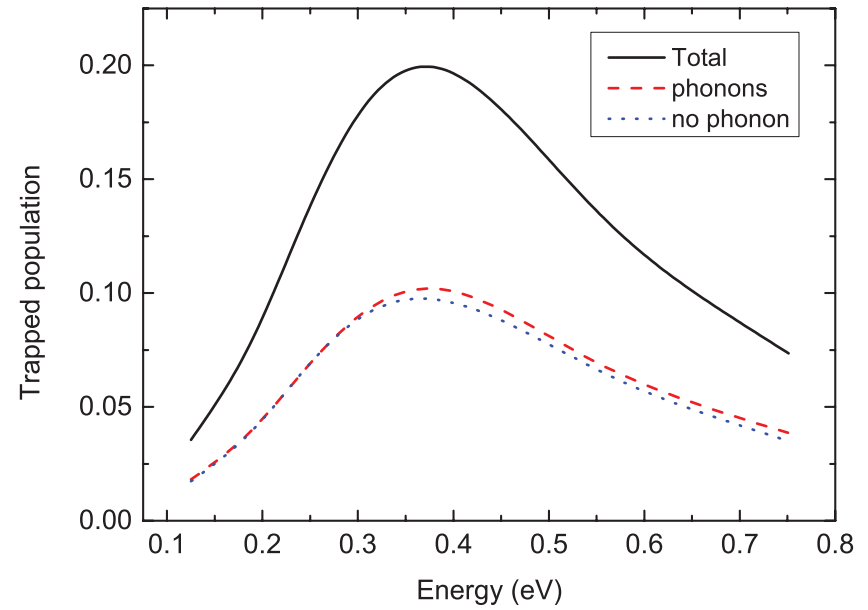

FIG. 5. Total trapped populations as a function of the mean energy of the wavepacket. Trapped population is the one in the chemisorption well $z<1.85 \AA$ at time 61 fs. Dotted and dashed lines: respective contributions of the $\lambda=0$ ("no phonon") and $\lambda= \pm 1$ ("phonons") components of the wavepacket. "Total" is the sum of both contributions. Surface temperature is $300 \mathrm{~K}$.

than the $n=1$ resonances by the large band wavepacket describing the collision. As a result, the $n=1$ states are the main contributors to trapping.

Finally, trapped populations at $300 \mathrm{~K}$ surface temperature are plotted as a function of the wavepacket mean energy of the incoming hydrogen on Fig. 5 for different surface states. The trapped probability is maximum at $0.38 \mathrm{eV}$, there is an energy threshold due to the dynamical barrier to chemisorption, which results from the addition of the saddle point energy on the PES and of the zero point vibrational energy at the transition state. Above $0.38 \mathrm{eV}$, trapping probabilities decrease with energy as a result of the reduced interaction time between faster hydrogen and surface.

\section{Discussion}

We first relate our results to the experimental ones of Refs. 20 and 21, performed for HOPG and graphene surfaces, at temperatures $150 \mathrm{~K}$ and $300 \mathrm{~K}$ with $\mathrm{H}$ atom mean kinetic energy of nearly $0.3 \mathrm{eV}$ and $0.4 \mathrm{eV}$, respectively. HOPG or graphene surfaces are exposed to incrementally increasing doses of hydrogen, and hydrogen coverage is obtained as a function of this dose from thermal desorption spectroscopy or from XPS on the $\mathrm{C} 1 \mathrm{~s}$ core level measurements. Comparable saturation coverages of $40 \% \pm 20 \%$ on HOPG and $25 \%$ for graphene are obtained for both experiments. This coverage value results from the competition between the adsorption and desorption/abstraction processes (hydrogen recombination in Ref. 21). If $P_{a d s}$ and $P_{H_{2}}$ are the corresponding probabilities, then a $25 \%$ saturation coverage implies: $P_{H_{2}}=3 P_{a d s}$. Adsorption probabilities are obtained from the short time hydrogen coverage kinetics. Indeed, coverage increases linearly at short times and the rate of increase is directly proportional to the sticking probability. Two measured sticking probabilities at low coverage differing by one order of magnitude: $P_{a d s}$ $=40 \% \pm 20 \%$ and $P_{a d s}=3.6 \%$ were obtained for $\mathrm{HOPG}^{20}$ and graphene. ${ }^{21}$ Interestingly, our result $P_{\text {ads }}=20 \%$ stands 
right in between the two measured quantities. It should be emphasized however that our result pertains to trapping rather than to sticking. But since our calculation shows that excitation of one phonon is a fast process, we expect that our trapping probability should overestimate only moderately the sticking one. This should be confirmed however by further investigations.

The large difference between the two measured sticking probabilities should be related to differences in experimental conditions. Interestingly, the smallest sticking probability is obtained in the experiment performed at the highest surface temperature. In our model, sticking increases with surface temperature: the coupling term for a giving phonon excitation increases with its occupation number (see Eq. (8)), which grows with surface temperature. This trend is expected to be moderate, however. It was shown in Ref. 16 that a surface temperature increase from $10 \mathrm{~K}$ to $300 \mathrm{~K}$, much larger than the present one, induces a moderate sticking probability increase, a factor of 2 in the case of physisorption. The decrease of the sticking probability by one order of magnitude when increasing surface temperature from $150 \mathrm{~K}$ to $300 \mathrm{~K}$ in the experiments should therefore be related to changes in experimental parameters other than temperature. Differences in sample preparation from graphite to graphene are expected to play a key role.

Our results can also be compared with those of other models. Sha et al.trapping probability (Ref. 23, Fig. 5) reaches a maximum of nearly $10 \%$ for a surface at $300 \mathrm{~K}$ and an incident $\mathrm{H}$ kinetic energy of $0.35 \mathrm{eV}$. A rather similar trapping probability, with a maximum of $\approx 8 \%$ at $0.3 \mathrm{eV}$, was also obtained in a subsequent classical model. ${ }^{26}$ Trapping is related to the transient population of vibrational states of the $\mathrm{CH}$ group, which are included in the model of Ref. 23 and in the present one. Our trapping probability restricted to the $\lambda$ $=0$ subspace (no surface state change) is close to the one of Refs. 23 and 26. The fact that we obtain additional trapping in the subspace related to surface state change suggests that phonon coupling is more efficient in our model than in theirs. This can be related to strong differences in the way to take this coupling into account. By contrast, Morisset et al. ${ }^{24,25,27} \mathrm{ob}-$ tained larger trapping probabilities than ours, using a model where the whole surface relaxes adiabatically as hydrogen atom approaches. Their simplified one dimensional dynamics does not incorporate a description of trapping resonances, but uses a realistic description of phonons. Our treatment is an attempt to combine the realistic model of the $\mathrm{CH}$ system of Refs. 23 and 26 with the phonon model of Refs. 24, 25, and 27. This combination provides intermediate results between the smaller values of Refs. 23 and 26 and the larger ones of Refs. 24, 25, and 27.

\section{CONCLUSIONS}

We have presented in this paper an extension to the chemisorption regime of the formalism we had used to study physisorption of hydrogen on graphene. ${ }^{16,17}$ This involved incorporating in the system coupled to the bath the puckering carbon atom nearest to the incoming hydrogen atom. This formalism was used to compute trapping probabilities, these re- sults were discussed in the context of experimental results on graphene and graphite.

Although the present model includes improvements with respect to previous ones, it still relies on approximations which should be removed to obtain more quantitative results. One is the one phonon approximation which is unavoidable within the wavepacket formalism used here in order to limit computations within reasonable limits. Going beyond by including multi-phonon processes would require to switch to completely different formalisms based for instance on the propagation of the reduced density matrix. ${ }^{15,56-61}$ Another significant approximation is the reduction of the dimensionality of the problem by assuming a perpendicular approach of the $\mathrm{H}$ atom right on top of a $\mathrm{C}$ atom. Future work should improve the model of hydrogenation along these lines.

\section{ACKNOWLEDGMENTS}

B.L. is grateful to Dr. Sabine Morisset for useful discussions. This work has been supported by the Barrande programme of French-Czech cooperation (project 7AMB12FR026). F.K. expresses his thanks to the Operational Programme Research and Development for Innovations - European Regional Development Fund (project CZ.1.05/2.1.00/03.0058) and to the Operational Program Education for Competitiveness - European Social Fund (projects CZ.1.07/2.3.00/20.0017 and CZ.1.07/2.3.00/20.0058). Financial support was also received from the French Agence Nationale de la Recherche under Grant No. ANR-08-BLAN0047-05 and from the program "Molécules et grains: du laboratoire à l'univers" of the Midi-Pyrénées Observatory.

${ }^{1}$ J. Roth, E. Vietzke, and A. A. Haasz, Suppl. to Nucl. Fusion 1, 63 (1991).

${ }^{2}$ J. Roth, E. Tsitrone, and A. Loarte, J. Phys.: Conf. Ser. 100, 062003 (2008).

${ }^{3}$ R. J. Gould and E. E. Salpeter, Astrophys. J. 138, 393 (1963).

${ }^{4}$ V. LePage, T. P. Snow, and V. M. Bierbaum, Astrophys. J. 704, 274 (2009).

${ }^{5}$ G. Vidali, Chem. Rev. 113, 8762 (2013).

${ }^{6}$ A. Li, J. Phys.: Conf. Ser. 6, 229 (2005).

${ }^{7}$ S. Ryu, M. Y. Han, J. Maultzsch, T. F. Heinz, P. Kim, M. L. Steigerwald, and L. E. Brus, Nano Lett. 8, 4597 (2008).

${ }^{8}$ D. C. Elias, R. R. Nair, T. M. G. Mohiuddin, S. V. Morozov, P. Blake, M. P. Halsall, A. C. Ferrari, D. W. Boukhvalov, M. I. Katsnelson, A. K. Geim et al., Science 323, 610 (2009).

${ }^{9}$ J. E. Johns and M. C. Hersam, Acc. Chem. Res. 46, 77-86 (2013).

${ }^{10}$ F. Karlický, K. K. R. Datta, M. Otyepka, and R. Zbořil, ACS Nano 7, 6434 (2013).

${ }^{11}$ B. Lassagne and A. Bachtold, C. R. Phys. (2010) 11, 355 (2010).

${ }^{12}$ E. Ghio, L. Mattera, C. Salvo, F. Tommasini, and U. Valbusa, J. Chem. Phys. 73, 556 (1980).

${ }^{13}$ M. Bonfanti, R. Martinazzo, G. F. Tantardini, and A. Ponti, J. Phys. Chem. C 111, 5825 (2007).

${ }^{14}$ V. Buch, J. Chem. Phys. 91, 4974 (1989).

${ }^{15}$ Z. Medina and B. Jackson, J. Chem. Phys. 125, 224703 (2006).

${ }^{16}$ B. Lepetit, D. Lemoine, Z. Medina, and B. Jackson, J. Chem. Phys. 134, 114705 (2011).

${ }^{17}$ B. Lepetit and B. Jackson, Phys. Rev. Lett. 107, 236102 (2011).

${ }^{18}$ L. Jeloaica and V. Sidis, Chem. Phys. Lett. 300, 157 (1999).

${ }^{19}$ X. Sha and B. Jackson, Surf. Sci. 496, 318 (2002).

${ }^{20}$ T. Zecho, A. Güttler, X. Sha, B. Jackson, and J. Küppers, J. Chem. Phys. 117, 8486 (2002).

${ }^{21}$ D. Haberer, C. E. Giusca, Y. Wang, H. Sachdev, A. V. Fedorov, M. Farjam, S. A. Jafari, D. V. Vyalikh, D. Usachov, X. Liu et al., Adv. Mater. 23, 4497 (2011).

${ }^{22}$ A. Paris, N. Verbitskiy, A. Nefedov, Y. Wang, A. Fedorov, D. Haberer, M. Oehzelt, L. Petaccia, D. Usachov, D. Vyalikh et al., Adv. Funct. Mater. 23, 1628 (2013) 
${ }^{23}$ X. Sha, B. Jackson, D. Lemoine, and B. Lepetit, J. Chem. Phys. 122, 014709 (2005).

${ }^{24}$ S. Morisset and A. Allouche, J. Chem. Phys. 129, 024509 (2008).

${ }^{25}$ S. Morisset, Y. Ferro, and A. Allouche, Chem. Phys. Lett. 477, 225 (2009).

${ }^{26}$ J. Kerwin, X. Sha, and B. Jackson, J. Phys. Chem. B 110, 18811 (2006).

${ }^{27}$ S. Morisset, Y. Ferro, and A. Allouche, J. Chem. Phys. 133, 044508 (2010).

${ }^{28}$ S. Garashchuk, J. Jakowski, L. Wang, and B. G. Sumpter, J. Chem. Theory Comput. 9, 5221 (2013)

${ }^{29}$ M. D. Stiles, J. W. Wilkins, and M. Persson, Phys. Rev. B 34, 4490 (1986).

${ }^{30}$ B. Jackson, J. Chem. Phys. 97, 6792 (1992).

${ }^{31}$ J. P. Perdew, K. Burke, and M. Ernzerhof, Phys. Rev. Lett. 78, 1396 (1997).

${ }^{32}$ G. Kresse and J. Furthmüller, Phys. Rev. B 54, 11169 (1996).

${ }^{33}$ G. Kresse and D. Joubert, Phys. Rev. B 59, 1758 (1999).

${ }^{34}$ T. Aizawa, R. Souda, S. Otani, Y. Ishizawa, and C. Oshima, Phys. Rev. B 42, 11469 (1990).

${ }^{35}$ L. Wirtz and A. Rubio, Solid State Commun. 131, 141 (2004).

${ }^{36}$ R. Peierls, Ann. I.H.P.: Phys. Theor. 5, 177 (1935).

${ }^{37}$ J. C. Meyer, A. K. Geim, M. I. Katsnelson, K. S. Novoselov, T. J. Booth, and S. Roth, Nature (London) 446, 60 (2007).

${ }^{38}$ J. H. Seol, I. Jo, A. L. Moore, L. Lindsay, Z. H. Aitken, M. T. Pettes, X. Li, Z. Yao, R. Huang, D. Broido et al., Science 328, 213 (2010).

${ }^{39}$ M. Ishigami, J. H. Chen, W. G. Cullen, M. S. Fuhrer, and E. D. Williams, Nano Lett. 7, 1643 (2007).

${ }^{40}$ X. Sha, B. Jackson, and D. Lemoine, J. Chem. Phys. 116, 7158 (2002).

${ }^{41}$ Y. Ferro, F. Marinelli, and A. Allouche, J. Chem. Phys. 116, 8124 (2002).

${ }^{42}$ Y. Ferro, F. Marinelli, and A. Allouche, Chem. Phys. Lett. 368, 609 (2003).

${ }^{43}$ E. J. Duplock, M. Scheffler, and P. J. D. Lindan, Phys. Rev. Lett. 92, 225502 (2004).
${ }^{44}$ P. O. Lehtinen, A. S. Foster, Y. Ma, A. V. Krasheninnikov, and R. M. Nieminen, Phys. Rev. Lett. 93, 187202 (2004).

${ }^{45}$ L. Hornekær, E. Rauls, W. Xu, Z. Sljivancanin, R. Otero, I. Stensgaard, E. Lægsgaard, B. Hammer, and F. Besenbacher, Phys. Rev. Lett. 97, 186102 (2006).

${ }^{46}$ L. Chen, A. C. Cooper, G. P. Pez, and H. Cheng, J. Phys. Chem. C 111, 18995 (2007).

${ }^{47}$ T. Roman, W. A. Diño, H. Nakanishi, H. Kasai, T. Sugimoto, and K. Tange, Carbon 45, 218 (2007).

${ }^{48}$ J. Kervin and B. Jackson, J. Chem. Phys. 128, 084702 (2008).

${ }^{49}$ E. Rauls and L. Hornekær, Astrophys. J. 679, 531 (2008).

${ }^{50}$ S. Casolo, O. M. Løvvik, R. Martinazzo, and G. F. Tantardini, J. Chem. Phys. 130, 054704 (2009).

${ }^{51}$ G. M. Psofogiannakis and G. E. Froudakis, J. Phys. Chem. C 113, 14908 (2009).

${ }^{52}$ D. Bachellerie, M. Sizun, F. Aguillon, D. Teillet-Billy, N. Rougeau, and V. Sidis, Phys. Chem. Chem. Phys. 11, 2715 (2009).

${ }^{53}$ V. Ivanovskaya, A. Zobelli, D. Teillet-Billy, N. Rougeau, V. Sidis, and P. Briddon, Eur. Phys. J. B 76, 481 (2010).

${ }^{54}$ N. Rougeau, D. Teillet-Billy, and V. Sidis, Phys. Chem. Chem. Phys. 13, 17579 (2011).

${ }^{55}$ Y. Wang, H. J. Qian, K. Morokuma, and S. Irle, J. Phys. Chem. A 116, 7154 (2012).

${ }^{56}$ R. K. Wangness and F. Bloch, Phys. Rev. 89, 728 (1953).

${ }^{57}$ A. G. Redfield, IBM J. Res. Dev. 1, 19 (1957).

${ }^{58}$ A. G. Redfield, Adv. Magn. Reson. 1, 1 (1965).

${ }^{59}$ B. Jackson, Chem. Phys. Lett. 270, 484 (1997).

${ }^{60}$ B. Jackson, J. Chem. Phys. 108, 1131 (1998).

${ }^{61}$ Z. Medina and B. Jackson, J. Chem. Phys. 128, 114704 (2008). 